\title{
Investigation of Diffraction Condition and Convergent Probe Effects on Inelastic Mean Free Path Determination by Using a Cone-Shaped Silicon Crystal
}

Qiang Jin and Shixin Wang

Micron Technology, Inc. Boise, Idaho 83707

As an important parameter in foil thickness measurement by electron energy-loss spectroscopy (EELS), the inelastic mean free path $(\lambda)$ not only varies with the collection angle $(\beta)$ of scattered electrons, but also depends on diffraction conditions of crystalline materials and the convergent angle $(\alpha)$ of the incident electron beam. In general, $\lambda$ decreases with increasing $\beta$. However, details of $\alpha$ and dynamical diffraction effects on the variation of $\lambda$ are not very clear, though some work has been done on this topic $[1,2]$. For a diffraction condition on a zone-axis with many beams within the collection aperture, the intensity ratio of inelastic and elastic scattering in the acquired EEL spectrum, i.e., $\mathrm{I}_{\mathrm{in}} / \mathrm{I}_{\mathrm{el}}$, can be expressed as:

$$
\frac{\mathrm{I}_{\text {in }}}{\mathrm{I}_{\mathrm{el}}}=\frac{\mathrm{I}_{\text {in }}(0)}{\mathrm{I}_{\mathrm{el}}(0)} \cdot \frac{1+\sum_{\theta \neq 0} \omega(\theta)}{1+\sum_{\theta \neq 0} \mathrm{~b}(\theta)}
$$

where $I_{\text {in }}(0)$ and $I_{\text {el }}(0)$ are the inelastic scattering intensity and elastic scattering intensity contributed from around the transmitted beam. $\omega(\theta)$ and $b(\theta)$ represent the relative contributions to the inelastic scattering and elastic scattering, respectively, from around a diffracted beam with Bragg angle $\theta$. The intensity ratio, $\mathrm{I}_{\mathrm{in}} / \mathrm{I}_{\mathrm{el}}$, determines the value of $\lambda$.

Utilization of a cone-shaped Si crystal prepared by a focused ion beam (FIB) [3], as shown in Fig.1, can overcome the difficulty in examining the dynamical diffraction effect on $\lambda$. In the experiment, the cone axis was aligned to the A-tilt axis so that the sample thickness ( $t$ ) passing through the cone axis was always equal to the projected cross-section diameter $(\mathrm{w})$, regardless of the A-tilt. The effect of misalignment between the cone axis and the A-tilt axis on a sample thickness measurement can be estimated as $t=w / \sqrt{1-\sin ^{2} \delta \sin ^{2} \alpha^{\prime}}$, where $\delta$ is the angle between the two axes and $\alpha$ is the angle of A-tilt. This effect is negligible with careful alignment of the two axes and slight A-tilt.

As shown in Fig.2, three types of diffraction conditions, many-beam, two-beam, and singlebeam, were used in this study. To achieve these diffraction conditions, the A-tilts are $6^{\circ}, 3^{\circ}$, and $11^{\circ}$, respectively, with almost no B-tilt $\left(<2^{\circ}\right)$. To evaluate the dynamic diffraction effect, the convergent angle $\alpha=6.4 \mathrm{mrad}$ was used, while $\alpha=15 \mathrm{mrad}$ was used to study the effect of the convergent probe. For each $\alpha$, different values of collection angle $\beta$ from 4.6-95 mrad were used by varying the camera length and the spectrometer entrance aperture. All experiments were conducted at $200 \mathrm{kV}$ and the EEL spectra were acquired with a diffraction pattern on screen. As shown in Fig.3a, the effects of diffraction conditions on $\lambda$ are negligible at large collection angles. Even at low collection angles, they only make moderate differences (less than 6\%). These results suggest that the transmitted beam in Eq. (1) plays a dominant role in determining $\lambda$, regardless of diffraction conditions. On the other hand, the convergence of the probe only affects $\lambda$ by reducing its value when $\alpha>\beta$ (Fig.3b). For $\beta>\alpha, \alpha$ has no effect on $\lambda$. The value of $\lambda$ depends on $\beta$ only and starts to saturate at $\beta \approx 20 \mathrm{mrad}$. The results shown in Fig.3b support those reported previously [1]. 


\section{References}

[1] T. Malis, S.C. Cheng and R.F. Egerton, J. Elec. Microsc. Tech. 8(1988) 193.

[2] A. Nakafuji, Y. Murakami and D. Shindo, J. Elec. Microsc. 50(2001) 23.

[3] S. Wang, Microsc. Microanal. 12, Suppl. 2 (2006).
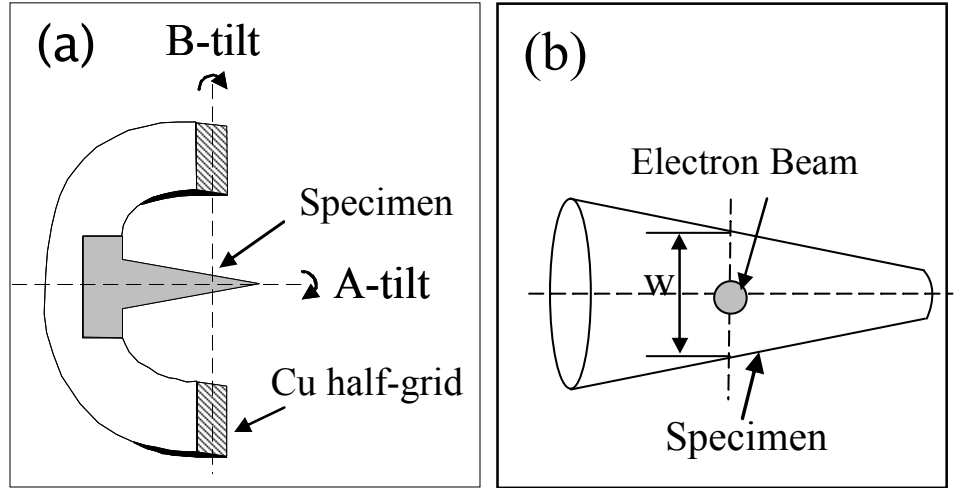

(c)

Fig.1 A cone-shaped specimen, (a) position alignment; (b) thickness measurement; (c) a TEM image.
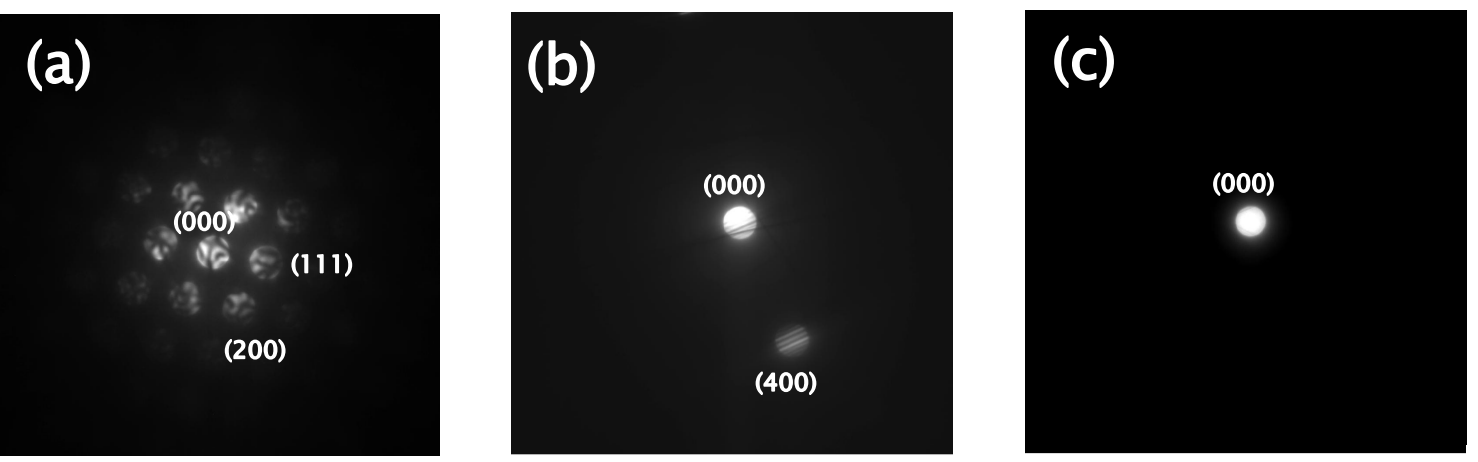

Fig.2 Diffraction conditions, (a) many-beam on (110) zone; (b) (400) two-beam; (c) (000) single-beam.
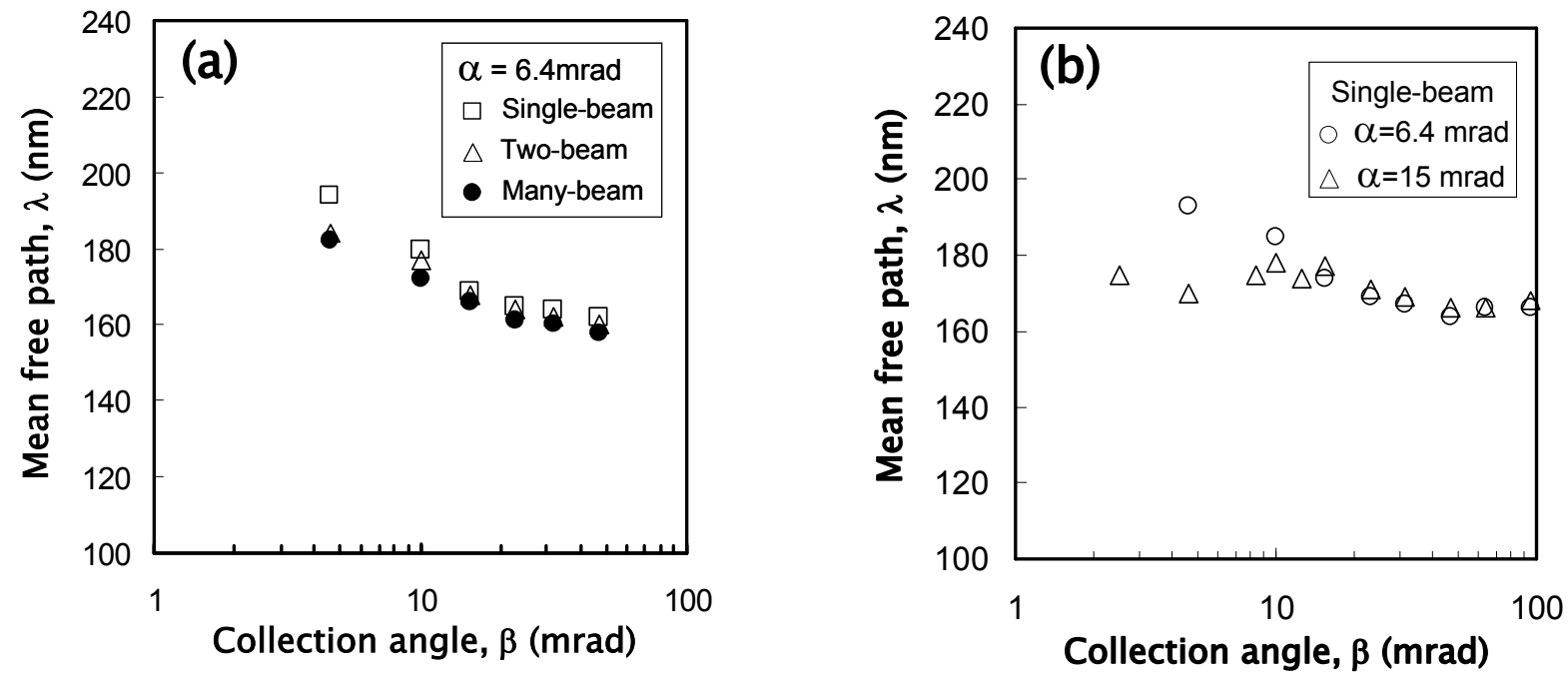

Fig. 3 Measured $\lambda$ values at sample thickness $t=450 \mathrm{~nm}$ with different collectionangles, (a) effect of diffraction condition; (b) effect of convergent probe. 